\title{
VELOCITY DISTRIBUTION OF FRAGMENTS IN COLLISIONAL BREAKUP
}

\author{
A. NAKAMURA and A. FUJIWARA \\ Dept. of Physics, Kyoto University \\ Kitashirakawa \\ Kyoto \\ 606 Japan
}

ABSTRACT. One of the key outcomes of collisional disruptions to the dust system is the velocity distribution of fragments. A series of laboratory impact experiments were carried out to obtain the massvelocity and the position-velocity relation of the fragments by taking movie films, and films for tow impacts were completely analyzed.

\section{INTRODUCTION}

Direct collisional process between solar system solid bodies ranging from dusts to planets is one of the major source of the interplanetary dust particles. The key outcomes of a collisional disruption to understand the evolution of the dust system is the velocity distribution as well as the size distribution of the fragments. The latter has been eagerly investigated experimentally and the sufficient amount of data afford to be compared with numerical simulations, while little experimental data about the motion of the individual fragments were obtained, although wide range of the fragment velocity was obtained from a cratering experiment against natural rock (Gault and Heitowit 1963).

Laboratory impact simulations were carried out to determine the velocity distribution of the fragments employing the popular highspeed photographic technique and an image processing system introduced in this field for the first time (Nakamura and Fujiwara 1990a).

\section{METHODS}

Here the experimental and the analytical procedures are outlined. The details are described in our other article (Nakamura and Fujiwara 1990a).

Spherical nylon projectiles of $0.70 \mathrm{~cm}$ in diameter were accelerated up to $3 \sim 4 \mathrm{~km} / \mathrm{sec}$ by a two-stage light-gas gun horizontally

A.C. Levasseur-Regourd and H. Hasegawa (eds.), Origin and Evolution of Interplanetary Dust, 379-382. (1) 1991 Kluwer Academic Publishers, Printed in Japan. 
aimed at basalt and alumina spheres of $6.0 \mathrm{~cm}$ in diameter. The incident angle of the projectile was changed in the plane including the trajectory and parallel to the floor. High-speed framing cameras were used at the framing rate of $6,000 \mathrm{frame} / \mathrm{sec}$, and the pairs of film records taken from two orthogonal directions to the trajectory, parallel (side-view film) and perpendicular (top-view film) to the floor, respectively, were dubbed into a video tape frame by frame. These two dimensional (2-D) video pictures are digitalized by an image processor linked to a personal computer and analyzed to get the position and the size of the 2-D images of the fragments.

Table 1 shows the precise conditions of the experiments for which we got the final result of the velocity distribution. Hundreds of fragments were observed for each impact.

TABLE 1. Experiment conditions

$\begin{array}{ll}\text { Target diameter } & =6.0(\mathrm{~cm}) \\ \text { Projectile diameter } & =0.70(\mathrm{~cm}) \\ \text { Projectile mass } & =0.21(\mathrm{~g}) \\ \text { Framing rate } & =6,000(\mathrm{frame} / \mathrm{sec})\end{array}$

\begin{tabular}{lccc}
\hline & Target & Projectile & Incident angle \\
mass $(\mathrm{g})$ & velocity $\left(\times 10^{5} \mathrm{~cm} / \mathrm{sec}\right)$ & to the surface \\
Alumina target & 395.7 & 4.00 & $60^{\circ}$ \\
Basalt target & 303.2 & 3.20 & $60^{\circ}$ \\
\hline
\end{tabular}

\section{RESULTS AND DISCUSSION}

The 2-D velocity and the initial position of each fragment were calculated by the least squares fit to its positions on the successive frames. The resulting $2-D$ velocity vectors are shown in Fig. 1 . The most prominent difference between the alumina and the basalt targets are the velocity profile of the fragments from the antipodal site. In the case of the alumina target, the antipodal fragments locally become smaller than those of the neighborhood. This antipodal character, higher in velocity and smaller in size, is also observed at the other three normal shots to the similar alumina targets, while the basalt fragments at the antipodes are larger and slower regardless of the incident angle $\left(90^{\circ} \sim 30^{\circ}\right.$ to the surface). Which physical property causes the difference between both targets has not been explained yet. The general axial symmetry around the normal at the impact site is observed for both targets, which probably suggests that the fragments we observed play minor role as the carrier of the incident angular momentum. This speculation is reinforced by the following discussion about the energy partitioning.

Scores of recovered fragments were identified with the 2-D images and empirical power law relations between the image size and the mass for both alumina and basalt cases were derived. The mass of the rest of all fragments were estimated by using the relations. Some of the 
(a)

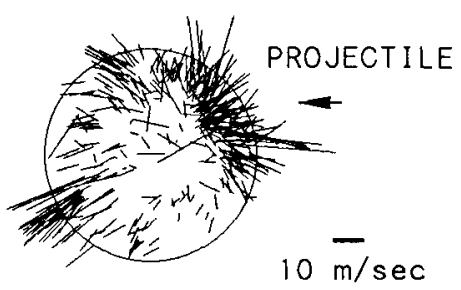

(b)

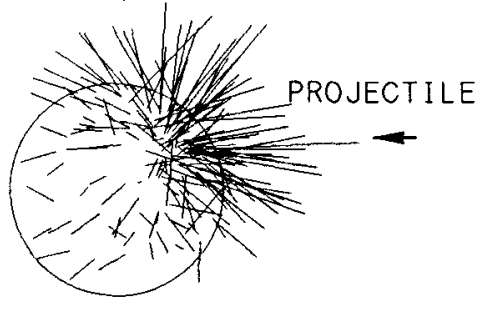

Figure 1. 2-D velocity vectors projected to the plane parallel to the floor of fragments from the alumina(a) target and the basalt(b) target listed in the Table 1 . The arrowheads are omitted.
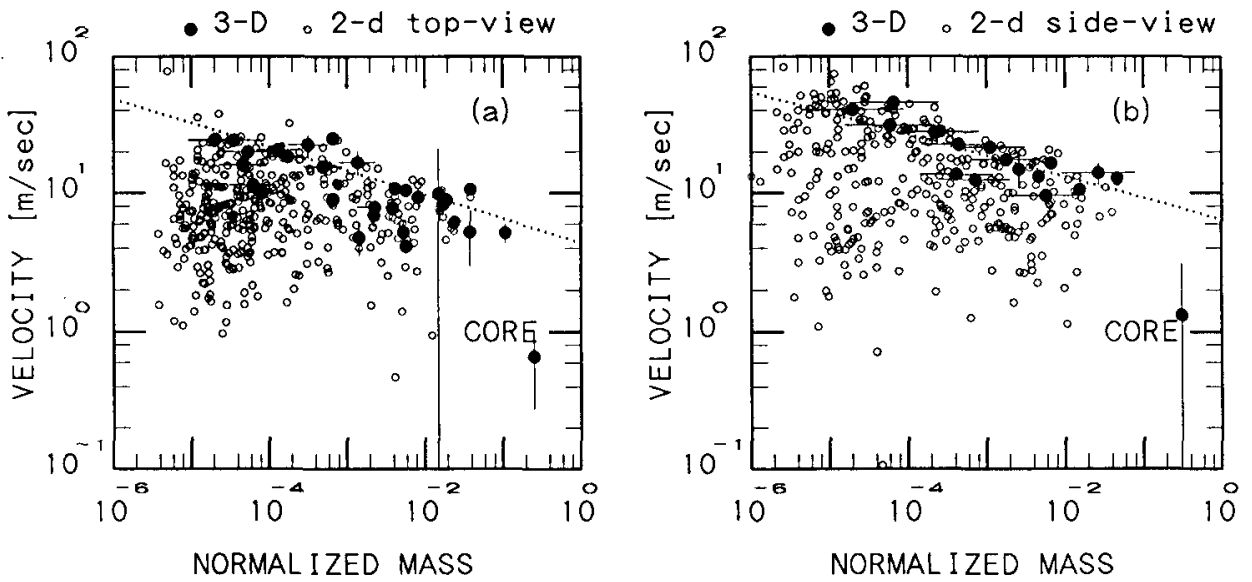

Figure 2. Mass-velocity distribution of fragments from the alumina target (a) and the basalt target (b) and the $3 \sigma$ error bars associated with the least squares fits determining the mass from the size and the velocity from the position of the fragments. The dashed lines represent the least squares fits to the data of the $3-\mathrm{D}$ velocity excluding the largest fragments. Roughly, the slopes are both $-1 / 6$.

fragments seen in the top-view film could be identified as the same fragments seen in the side-view. Figure 2 shows the 2-D and 3-D massvelocity distribution of fragments. The slopes of the upper boundary of the 2-D velocity distribution agree reasonably with those of dashed lines obtained from least squares fits to the $3-D$ velocity data except those of largest fragments (called 'core'). The 3-D velocity of the largest fragments apparently deviate from the dashed lines. This deviation would be explained by the different sites they were created or different processes they were suffered. The largest fragments are spherical ones left at the center of the targets, while almost all the 
other fragments we got the 3-D velocity are from the surface of the targets created by the tensile stress generated at the surface. The slope of the fitted lines are both about $-1 / 6$ indicating the translational kinetic energy of individual fragments are proportional to the (mass) ${ }^{2,3}$, i.e. the same dimension as the surface area of the fragments.

Total translational kinetic energy partitioned into fragments heavier than $10 \mathrm{mg}$ was estimated from the above 3-D mass-velocity distributions and the mass distribution. Only one or two percent of the initial kinetic energy was partitioned to those larger fragments at both impacts. The rotational energy of the spinning motion of these heavier fragments could not be larger than the translational kinetic energy regarding, though insufficient, the experimental data(Fujiwara 1987; Nakamura and Fujiwara 1990b). Major fraction of the incident energy would be expended in the various energy mode, such as kinetic energy and comminution energy, of fine fragments from near the impact sites. The incident angular momentum is expected to be also carried away by these fine ejecta.

\section{CONCLUSION}

The employment of the image processor bring us a relatively systematic analysing method, however, there are much inconvenience which prevent us from a swift analysing and more improvements are desirable.

Although the present analysis is limited to the two impacts, the resulting velocity distributions are still fairly suggestive. More experimental data concerning to the motion of the fragments are required to confirm the $-1 / 6$ power law of the velocity distribution and the speculation that the kinetic energy is proportional to the surface area, to define the physical conditions where the $-1 / 6$ power law holds, or to find out that the results here are only the stochastic products, and eventually to understand the grinding process between solid bodies ranging from dusts to planets.

\section{REFERENCES}

Gault, D.E. and Heitowit, E.D.(1963) 'The partition of energy for hypervelocity impact craters formed in rock', Proc. 6th Hypervelocity Impact Symp. Vol 2 (Cleveland. OH: Firestone Rubber Co.), pp 419-456.

Fujiwara, A. (1987) 'Energy partition into translational and rotational motion of fragments in catastrophic disruption by impact:An experiment and asteroid cases', Icarus 48, 329-334.

Nakamura, A. and Fujiwara, A. (1990a) 'Velocity distribution of fragments formed in simulated collisional disruption', Submitted to I carus.

Nakamura, A. and Fujiwara, A. (1990b) 'Rotational motion of fragments from hyper-velocity impact experiments', Proc. 23th ISAS Lunar and Planet Symp. 